\title{
Haptoglobin 1-1 is Associated with Larger Carotid Plaque Volume Among Elderly with Type 2 Diabetes: The IDCD Study.
}

Chen Botvin ( $\square$ chen.botvin@gmail.com )

Tel Aviv University https://orcid.org/0000-0002-4894-9659

\section{Salo Haratz}

Assuta Ashdod Hospital

Ramit Ravona-Springer

Sheba Medical Center at Tel Hashomer

Anthony Heymann

Maccabi Healthcare Services

Andrew Levy

Technion Israel Institute of Technology Ruth and Bruce Rappaport Faculty of Medicine

Michal Schnaider Beeri

Icahn School of Medicine at Mount Sinai

David Tanne

Rambam Health Care Campus

Original investigation

Keywords: IDCD, haptoglobin, carotid plaque volume, carotid atherosclerosis, diabetes

Posted Date: June 22nd, 2020

DOI: https://doi.org/10.21203/rs.3.rs-35204/v1

License: (c) (i) This work is licensed under a Creative Commons Attribution 4.0 International License.

Read Full License 


\section{Abstract}

Background and aims: The haptoglobin ( $\mathrm{Hp}$ ) genotype (Hp 1-1, 1-2, or 2-2) is associated with type 2 diabetes complications. The Hp2-2 genotype has been linked to elevated CHD risk while the 1-1 genotype has been recently related to cerebral small vessel disease. This study investigated whether Hp phenotype is associated with carotid atherosclerotic structure and function among type 2 diabetes elderly patients participating in the Israel Diabetes and Cognitive Decline (IDCD) study.

Methods: IDCD participants ( $\mathrm{n}=472)$ underwent a battery of carotid atherosclerosis ultrasound assessments with measurements of carotid intima media thickness (cIMT), distensibility, elastography, and carotid plaque volume. Ordinal regression models adjusting for demographic and cardiovascular risk factors assessed the associations of categorical measures of carotid plaque volume with Hp phenotype . Logistic regression models were used to assess the association of Hp phenotype with impaired clMT, carotid distensibility and elastography.

Results: Participants averaged $76.4 \pm 4.4$ years old, $42 \%$ were female, mean $\mathrm{HbA} 1 \mathrm{c}$ was $6.7 \pm 0.7 \%(50 \pm 7.7$ $\mathrm{mmol} / \mathrm{mol})$. Hp phenotype distribution was 46 (10.4\%) Hp 1-1, 186 (40.7\%) Hp 2-1 and 209 (45.7\%) Hp 22 carriers. Carotid plaque volume was categorized into 4 groups: including no plaque $(n=128)$ and tertiles of plaque volume: small plaque (volume $\leq 122 \mathrm{~mm}^{3}, \mathrm{n}=110$ ), medium plaque (volume $122-273 \mathrm{~mm}^{3}$, $\mathrm{n}=106$ ) and large plaque (volume $\geq 274 \mathrm{~mm}^{3}, \mathrm{n}=104$ ). The prevalence of $\mathrm{Hp} \mathrm{1-1}$ phenotype increased with greater carotid plaque volume $(6.5 \%, 10.6 \%, 8.8 \%$ and $16.5 \%$ in the no, small, medium, and large plaque groups respectively; $\mathrm{p}=0.027$ ). Compared to $\mathrm{Hp}-1$ carriers, non-carriers (i.e. $\mathrm{Hp} \mathrm{2-1}$ and $\mathrm{Hp} \mathrm{2-2}$ ) had approximately $30 \%$ lower risk for large carotid plaque volume, as estimated using ordinal regression (OR: $0.713[0.496,1.03], 0.694[0.484,0.997]$ for $\mathrm{Hp} 2-1, \mathrm{Hp} 2-2$ respectively). No associations were found between $\mathrm{Hp}$ phenotype and level of carotid stenosis, cIMT, carotid distensibility and carotid elastography.

Conclusion: Our findings suggest that among non-demented elderly patients with type 2 diabetes, the Haptoglobin 1-1 phenotype is associated with greater carotid atherosclerosis. These findings add evidence for the involvement of the Hp 1-1 phenotype in the pathophysiology of cerebrovascular disease.

\section{Background}

Haptoglobin (Hp) is an acute phase protein that binds to freely circulating hemoglobin. $\mathrm{Hp}$ is encoded from the haptoglobin gene which exists as two alleles, $\mathrm{Hp}-1$ and $\mathrm{Hp}-2$. The function of haptoglobin is primarily to determine the fate of hemoglobin released from red blood cells after either intravascular or extravascular hemolysis. There are functional differences between the $\mathrm{Hp} 1$ and $\mathrm{Hp} 2$ protein products in protecting against hemoglobin-driven oxidative stress that appear to have important clinical significance ${ }^{1}$.

Hp genotype has been consistently associated with vascular diabetes complications, both acute cardiovascular events and vascular deterioration. Type 2 diabetes patients with $\mathrm{Hp}$ 2-2 phenotype are at 
greater risk of increased carotid intima media thickness (cIMT) ${ }^{2,3}$, restenosis following stenting ${ }^{4}$ and cardiovascular disease (CVD) $)^{5,6}$ and mortality ${ }^{2}$. In contrast, a lower prevalence of CVD in type 2 diabetes patients with Hp 2-1 and Hp 2-2 genotype compared with Hp 1-1 genotype has been reported ${ }^{7}$.

Moreover, in recent years there is a growing line of evidence for the association of $\mathrm{Hp} \mathrm{1-1}$ with cerebrovascular disease ${ }^{8,9}$. In a case-control study $\mathrm{Hp}-1$ frequency was higher among patients with first symptomatic lacunar stroke due to small vessel disease compared with controls ${ }^{10}$. Increase in the number of $\mathrm{Hp}-1$ alleles was associated with a greater volume of cerebral white matter hyperintensities, a marker for cerebral small vessel disease, in patients with hypertension ${ }^{9}$ and with type 1 diabetes $^{8}$. On the other hand patients with type 2 diabetes and Hp 2-2 phenotype were at higher risk for greater carotid intima media thickness (cIMT) and for unstable carotid plaque ${ }^{3,5}$.

Recent ultrasound developments have allowed to investigate carotid plaque volume with high reliability and repeatability ${ }^{11,12}$. Carotid plaque volume was demonstrated as a superior marker compared to cIMT and to carotid plaque area for prediction of $C V D^{13}$ and cardiovascular events ${ }^{14,15}$. Additional innovative markers for carotid atherosclerosis are carotid distensibility for the measurement of carotid artery stiffness and carotid elastography for measurement of carotid artery wall properties. It was demonstrated that carotid artery stiffness, as determined by distensibility coefficient, is associated with atherosclerosis and its outcome such as stroke, TIA and CVD ${ }^{16,17}$. In this study, we used a broad ultrasound assessment including 3D sonography-carotid plaque volume, a marker of the extent of carotid atherosclerosis, clMT, carotid distensibility and strain ratio elastography for the evaluation of the association between $\mathrm{Hp}$ phenotype and carotid atherosclerosis markers among elderly cognitively normal type 2 diabetes patients from the Israel Diabetes and Cognitive Decline (IDCD) study ${ }^{18}$.

\section{Methods}

\section{Study population}

Participants are from the IDCD study, which investigates the effects of long-term type 2 diabetes-related characteristics on cognitive decline. The IDCD study design has been previously described in detail ${ }^{18}$. Briefly, the IDCD recruited community-dwelling elderly individuals with type 2 diabetes (65+ years old) living in central Israel, from approximately 11,000 clients enrolled in the diabetes registry of the Maccabi Healthcare Services (MHS). MHS is the second largest health maintenance organization (HMO), treating a representative cross-section of 2 million Israeli citizens. The MHS diabetes registry was established in 1998 to facilitate diabetes management and to improve treatment. Any of the following criteria were sufficient for enrolment into the registry: (1) HbA1c >7.25\% (55.7 mmol $/ \mathrm{mol}$ ); (2) glucose $>11.10 \mathrm{mmol} / \mathrm{l}$ on two exams more than 3 months apart; (3) purchase of diabetic medication twice within 3 months supported by an $\mathrm{HbA} 1 \mathrm{c}>6.5 \%(47.5 \mathrm{mmol} / \mathrm{mol})$ or glucose $>6.94 \mathrm{mmol} / \mathrm{l}$ within half a year; (4) diagnosis of type 2 diabetes (ICD-9 code ${ }^{19}$ by a general practitioner, internist, endocrinologist, ophthalmologist or type 2 diabetes advisor, supported by an $\mathrm{HbA} 1 \mathrm{c}>6.5 \%(47.5 \mathrm{mmol} / \mathrm{mol})$ or glucose $>6.94 \mathrm{mmol} / \mathrm{l}$ within 
half a year. These criteria have been validated by 20 physicians in the MHS against their own practice

records ${ }^{20}$. IDCD inclusion criteria were having type 2 diabetes; normal cognition at entry; being free of any neurological (e.g., Parkinson's disease, stroke), psychiatric (e.g., schizophrenia) or other diseases (e.g., alcohol or drug abuse) that might affect cognition; Hebrew fluency, and having an informant. Participants were assessed by a physician experienced in assessment and diagnosis of dementia, and by a neuropsychologist, who administered a neuropsychological battery. Carotid atherosclerosis was assessed during the IDCD 36 months follow up. Four hundred seventy-two IDCD participants underwent the carotid US assessments and had complete data on $\mathrm{Hp}$ and sociodemographic and cardiovascular variables.

\section{Covariates}

Data on risk factors and possible confounders were obtained using two methods: the Maccabi Diabetes Registry and data collection during the baseline visit of the IDCD cohort. Variables available through the Diabetes Registry were computed as the average of all the measurements done in Maccabi since entry to the Diabetes Registry and included time in the T2D registry (a good proxy of duration of T2D) ${ }^{21}, \mathrm{BMI}$, $\mathrm{HbA1c}$, fasting glucose, total, HDL and LDL cholesterol, triglycerides, vitamin B-12, vitamin D, and folic acid levels. Systolic and diastolic blood pressure was measured during the carotid US examination. During the baseline visit of the IDCD study blood samples were obtained to evaluate CRP, IL- 6 and the phenotype of APOE and haptoglobin.

\section{Ultrasound assessment procedures}

All examinations were performed at the Department of Neurology, Sheba Medical Center by one of 2 qualified and experienced ultrasound technicians, after obtaining informed consent. Subjects were placed in a supine position and rested for 5 minutes prior to assessing their vital signs. Carotid Ultrasound Doppler was performed using the premium EPIQ 7 US system (Philips, Netherlands). The following indices of carotid stiffness and atherosclerosis were assessed:

Carotid intima-media thickness (cIMT): IMT is defined as the distance between the media-adventitia interface and the lumen-intima interface. Measurements were performed bilaterally at the far wall of the common carotid artery (CCA) $1.0 \mathrm{~cm}$ proximal to the carotid bifurcation. The mean value of computerbased points was used. For each individual, CIMT was determined as the average of 3 measurements for each artery, as was automatically computed by the QLAB software (Philips, Netherlands).

3-D Carotid plaque volume: Patients with detectable plaques were assessed for plaque volume. In standard optimized mode, using the mechanic volumetric VL13-5 broadband linear array transducer, 3D plaque scanning volume data were obtained automatically. For each volume approximately 250 single transverse images (frames) were obtained with an interval of $0.15 \mathrm{~mm}$. Plaque volume was automatically calculated using the Vascular Plaque Quantification (VPQ) module (QLAB software), after selecting the beginning and ending frame and selecting at least one key frame within the plaque region. 
Carotid Distensibility: Following static B-mode real-time imaging from a longitudinal section of the CCA after 5 minutes of rest, dynamic CINE looped M-mode images of consecutive cardiac cycles were stored for later offline analysis. Distensibility was assessed using the distension of both CCAs, measuring the change in diameter in systole relative to diastolic during the cardiac cycle. The vessel lumen diameter was assessed from the from the near wall to the far wall of the CCA. The maximal systolic lumen diameter was determined visually and from the R-wave of the ECG-recording and the minimal lumen diameter was used for the diastolic diameter. The end-diastolic diameter (Dd), the absolute stroke change in diameter during systole $(\Delta \mathrm{A})$, and the relative stroke change in diameter $(\Delta \mathrm{A} / \mathrm{Dd})$ were computed as the mean of 10 cardiac cycles of one successive recording. Blood pressure was measured before and after the measurement session and pulse pressure $(\Delta P)$ was defined as the difference between the systolic and diastolic blood pressure. The cross-sectional arterial wall distensibility coefficient was calculated according to the following equation:

Cross sectional distensibility coefficient $(D C)=1000 * \frac{\Delta A}{A} * \Delta P\left[\mathrm{kPa}^{-1}\right]$

Carotid Elastography: In the B-mode display, a midsection of straight CCA in longitudinal plane is chosen. The shear wave elastographic mode was activated to show paired images of B-mode and elastography at the same time. The probe was handed with standardized pressure in the $2^{\text {nd }}-4^{\text {th }}$ quintile of the linear pressure scale, as seen using standardized real-time measurement displayed on a linear scale.

Elastographic images are displayed with different color mapping for the softest, intermediate and hardest components, according to the different levels of strain. On a representative static image, the relative strain ratio (SR), between blood to carotid arterial wall were measured. The first region of interest (ROI) for the arterial wall strain was manually placed at the midpoint of posterior wall of displayed carotid artery. The second ROI for the blood strain was placed at the center of arterial lumen. SR was calculated automatically by dividing strain value of the blood by that of carotid arterial wall, using the QLAB software. Measurement were performed during 10 heart beats and an average of 3 images, preferably consecutive, were used as the elastography index.

\section{Haptoglobin genotyping:}

Blood serum samples for Hp phenotype were drawn from subjects during the baseline visit. Genotyping was determined using polyacrylamide gel electrophoresis method according to established methods ${ }^{22}$.

\section{Statistical methods}

All variables were reviewed for abnormal values, to assess skewedness and outliers. Characteristics of study participants were compared using independent-samples T-test, Wilcoxon rank-test and ANOVA, as appropriate for continuous variables, and $\chi^{2}$ test for categorical variables.

Total burden of carotid plaque volume was computed as the sum of plaques volume in the right and left carotid arteries. Due to a non-normal distribution of carotid plaque volume, it was a-priori categorized into 
4 groups: no plaque group, and tertiles of the plaque volume: small plaque (volume $\leq 122 \mathrm{~mm}^{3}$ ) medium plaque (volume 122.1-271 $\mathrm{mm}^{3}$ ) and large plaque (volume $>271 \mathrm{~mm}^{3}$ ). Impaired clMT, carotid distensibility and elastography were defined as the worst quartile. cIMT worst quartile was determined as higher or equal to $0.9 \mathrm{~mm}$, carotid distensibility was determined as the lower or equal to $13.03\left[\mathrm{kPa}^{-3}\right]$ and carotid elastography was determined as higher or equal to $0.925 \mathrm{SR}$.

Haptoglobin phenotype was also used as an ordinal variable where 1-1 phenotype was the reference group for the 2-1 and 2-2 phenotypes. Mantel-Haenszel linear by linear association was used for the trend analysis between carotid plaque volume and haptoglobin phenotype. ANCOVA was used to estimate the association of cIMT, carotid distensibility and elastography with Hp phenotype. Ordinal logistic regression was used to estimate the odds ratio (OR) and 95\% confidence interval (Cl) for the association between carotid plaque volume with $\mathrm{Hp}$ phenotype. Binary logistic regression was used to estimate the odds ratio (OR) and $95 \%$ confidence interval $(\mathrm{Cl})$ for the association between impaired cIMT, carotid distensibility and elastography with $\mathrm{Hp}$ phenotype. Primary covariates in all analyses were age and gender as they are strongly associated with both predictors and outcomes. Secondary covariates were LDL cholesterol, triglycerides, II-6, eGFR, diabetes treatment (none, oral hypoglycemic, insulin, oral hypoglycemic+insulin), duration of diabetes and smoking. Statistical analysis was performed using SPSS software version 24.

\section{Results}

\section{Patient characteristics}

Subjects were $42 \%$ female, mean age of $76.4 \pm 4.4$ years, mean $\mathrm{HbA} 1 \mathrm{c}$ of $6.7 \pm 0.7 \%(50 \pm 7.7 \mathrm{mmol} / \mathrm{mol})$.

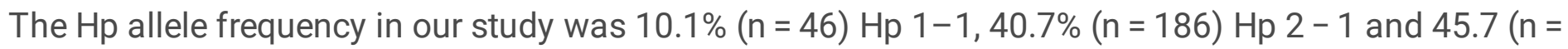

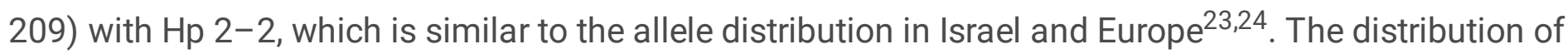
the haptoglobin phenotypes in this cohort was consistent with the Hardy-Weinberg equation ( $\mathrm{Hp} 1$ frequency $=0.3099$, Hp 2 frequency $=0.6901$ ). As shown in Table 1, there were no significant differences between the Hp phenotypes in any of the sociodemographic or cardiovascular characteristics. Level of stenosis, IMT, carotid distensibility and carotid elastography in the carotid artery bifurcation did not differ among the $3 \mathrm{Hp}$ phenotypes (Table 1). Plaque volume by the $3 \mathrm{Hp}$ phenotypes was $223.47 \pm 237.91 \mathrm{~mm}^{3}$, $171.69 \pm 204.68 \mathrm{~mm}^{3}$, and $152.23 \pm 166 \mathrm{~mm}^{3}$ for $\mathrm{Hp} 1-1, \mathrm{Hp} 2-1$ and $\mathrm{Hp} 2-2$ respectively $(p=0.073)$. 
Table 1

Baseline demographics, clinical characteristics and carotid atherosclerosis by $\mathrm{Hp}$ phenotype.

\begin{tabular}{|c|c|c|c|c|}
\hline & $\begin{array}{l}\text { Hp 1-1 } \\
(n=46)\end{array}$ & $\begin{array}{l}\text { Hp 1-2 } \\
(n=186)\end{array}$ & $\begin{array}{l}\text { Hp 2-2 } \\
(n=209)\end{array}$ & $\mathbf{P}$ \\
\hline Gender & $31(61.4 \%)$ & $111(59.7 \%)$ & $120(57.4 \%)$ & 0.773 \\
\hline Male & $15(32.6 \%)$ & $75(40.3 \%)$ & $89(42.6 \%)$ & \\
\hline \multicolumn{5}{|l|}{ Female } \\
\hline Age [years] & $72 \pm 4$ & $71 \pm 4$ & $71 \pm 4$ & 0.361 \\
\hline Years diagnosed & $9.54 \pm 4.59$ & $9.2 \pm 4.6$ & $9.87 \pm 4.27$ & 0.323 \\
\hline Mean $\mathrm{HbA} 1 \mathrm{c}[\%]$ & $6.66 \pm 0.77$ & $6.73 \pm 0.72$ & $6.62 \pm 0.7$ & 0.350 \\
\hline$(\mathrm{mmol} / \mathrm{mol})$ & $(49 \pm 7.9)$ & $(50 \pm 7.7)$ & $(49 \pm 7.7)$ & \\
\hline Triglycerides [mg/dL] & $136.8 \pm 50.53$ & $155.78 \pm 83.47$ & $158.66 \pm 57.98$ & 0.153 \\
\hline LDL cholesterol [mg/dL] & $104.35 \pm 21.88$ & $98.78 \pm 18.78$ & $101.95 \pm 21.45$ & 0.130 \\
\hline HDL cholesterol [mg/dL] & $47.13 \pm 9.88$ & $48.98 \pm 11.02$ & $48.18 \pm 11.2$ & 0.544 \\
\hline BMI $\left[\mathrm{kg} / \mathrm{cm}^{2}\right]$ & $28.35 \pm 5.67$ & $28.6 \pm 4.39$ & $28.51 \pm 4.18$ & 0.947 \\
\hline Systolic BP [mmHg] & $146 \pm 22$ & $141 \pm 19$ & $145 \pm 23$ & 0.184 \\
\hline Diastolic BP [mmHg] & $74 \pm 9$ & $73 \pm 9$ & $74 \pm 11$ & 0.768 \\
\hline eGFR $\left[\mathrm{ml}^{-1}{ }^{-1} \min ^{-1} * 1.73 \mathrm{~m}^{-2}\right]$ & $0(0 \%)$ & $7(4.0 \%)$ & $4(2 \%)$ & 0.221 \\
\hline$\leq 45$ & $7(15.6 \%)$ & $30(16.8 \%)$ & $38(19 \%)$ & \\
\hline $45-60$ & $38(84.4 \%)$ & $142(79.3 \%)$ & $158(79 \%)$ & \\
\hline \multicolumn{5}{|l|}{$\geq 60$} \\
\hline Diabetic Medications & $8(21.6 \%)$ & $20(13.1 \%)$ & $27(15.0 \%)$ & 0.737 \\
\hline No medication & $25(67.6 \%)$ & $116(75.8 \%)$ & $139(77.2 \%)$ & \\
\hline Oral medication & $4(10.8 \%)$ & $17(11.1 \%)$ & $14(17.8 \%)$ & \\
\hline \multicolumn{5}{|l|}{ Insulin } \\
\hline Smoking & $21(46.7 \%)$ & $66(36.1 \%)$ & $81(39.7 \%)$ & 0.225 \\
\hline Never smoked & $17(37.8 \%)$ & $98(58.6 \%)$ & $102(50 \%)$ & \\
\hline Smoked in the past & $7(15.6 \%)$ & 19 (10.4\%) & $21(10.3 \%)$ & \\
\hline Smoking & & & & \\
\hline
\end{tabular}




\begin{tabular}{|lllll|}
\hline & $\begin{array}{l}\text { Hp 1-1 } \\
(\mathbf{n = 4 6 )}\end{array}$ & $\begin{array}{l}\text { Hp 1-2 } \\
(\mathbf{n = 1 8 6})\end{array}$ & $\begin{array}{l}\text { Hp 2-2 } \\
(\mathbf{n = 2 0 9})\end{array}$ & P \\
\hline Level of stenosis & $4(9.1 \%)$ & $32(18 \%)$ & $36(17.9 \%)$ & 0.558 \\
No plaque & $27(61.4 \%)$ & $86(48.3 \%)$ & $99(49.3 \%)$ & \\
$<50 \%$ & $12(27.3 \%)$ & $55(30.9 \%)$ & $56(27.9 \%)$ & \\
$50 \%-69 \%$ & $1(2.3 \%)$ & $5(2.8 \%)$ & $10(5 \%)$ & \\
$>70 \%$ & & & & 0.073 \\
\hline Carotid plaque volume & $223.47 \pm 237.91$ & $171.69 \pm 204.68$ & $152.23 \pm 166$ & 0.792 \\
\hline Carotid plaque area [mm $\left.{ }^{2}\right]$ & $17.82 \pm 19.51$ & $17.31 \pm 18.75$ & $16.25 \pm 17.5$ & 0.768 \\
\hline Common carotid IMT [mm] & $0.82 \pm 0.16$ & $0.82 \pm 0.16$ & $0.81 \pm 0.14$ & 0.567 \\
\hline Carotid Distensibility [kPa $\left.{ }^{-3}\right]$ & $18.65 \pm 7.81$ & $17.67 \pm 7.31$ & $18.45 \pm 7.04$ & 0.907 \\
\hline Carotid Elastography [SR] & $0.75 \pm 0.28$ & $0.78 \pm 0.34$ & $0.77 \pm 0.29$ & \\
\hline
\end{tabular}

The distribution of Hp phenotype by carotid plaque volume groups is depicted in Table 2. The prevalence of $\mathrm{Hp} 1-1$ phenotype increased with carotid plaque volume group $(6.5 \%, 10.6 \%, 8.8 \%$ and $16.5 \%$ in the no, small, medium, and large plaque respectively). The prevalence of $\mathrm{Hp} 2-1$ did not change with the plaque volume group, and the prevalence of $\mathrm{Hp} 2-2$ decreased with the plaque volume group $(48.8 \%, 52.9 \%$, $45.1 \%$ and $43.7 \%$ in the no, small medium and large plaque respectively $(p=0.027$ by the MantelHaenszel linear by linear association).

Table 2

Distribution of plaque size groups by Hp phenotypes. P-value for trend was determined by MentelHeanzel linear by linear test

\begin{tabular}{|c|c|c|c|c|c|}
\hline \multirow[t]{2}{*}{ Subject group } & \multirow{2}{*}{$\begin{array}{l}\text { Sample } \\
\text { size }\end{array}$} & \multicolumn{3}{|c|}{ Haptoglobin phenotype } & \multirow[t]{2}{*}{ p-value } \\
\hline & & $1-1$ & $2-1$ & $2-2$ & \\
\hline No plaque, n(\%) & $N=123$ & $8(6.5 \%)$ & $55(44.7 \%)$ & $60(48.8 \%)$ & \multirow{4}{*}{$\begin{array}{l}\text { P for trend }= \\
0.027\end{array}$} \\
\hline Small plaque, $\mathrm{n}(\%)$ & $N=104$ & $\begin{array}{l}11 \\
(10.6 \%)\end{array}$ & $38(36.5 \%)$ & $55(52.9 \%)$ & \\
\hline $\begin{array}{l}\text { Medium plaque, } \\
\mathrm{n}(\%)\end{array}$ & $N=102$ & $9(8.8 \%)$ & $47(46.1 \%)$ & $46(45.1 \%)$ & \\
\hline Large plaque, n(\%) & $N=103$ & $\begin{array}{l}17 \\
(16.5 \%)\end{array}$ & $41(39.8 \%)$ & $45(43.7 \%)$ & \\
\hline Total, n(\%) & $N=488$ & $\begin{array}{l}45 \\
(10.4 \%)\end{array}$ & $\begin{array}{l}181 \\
(41.9 \%)\end{array}$ & $\begin{array}{l}206 \\
(47.7 \%)\end{array}$ & \\
\hline
\end{tabular}


Table 3

estimation of the relative odds of experiencing an increased carotid plaque group or impaired cIMT, carotid distensibility or elastography by ordinal logistic regression or binary logistic regression respectively, model 1: unadjusted, model 2: adjusted for age and gender, model 3 adjusted for age, gender, LDL cholesterol, triglycerides, II-6, eGFR, diabetes treatment, years of diabetes and smoking.

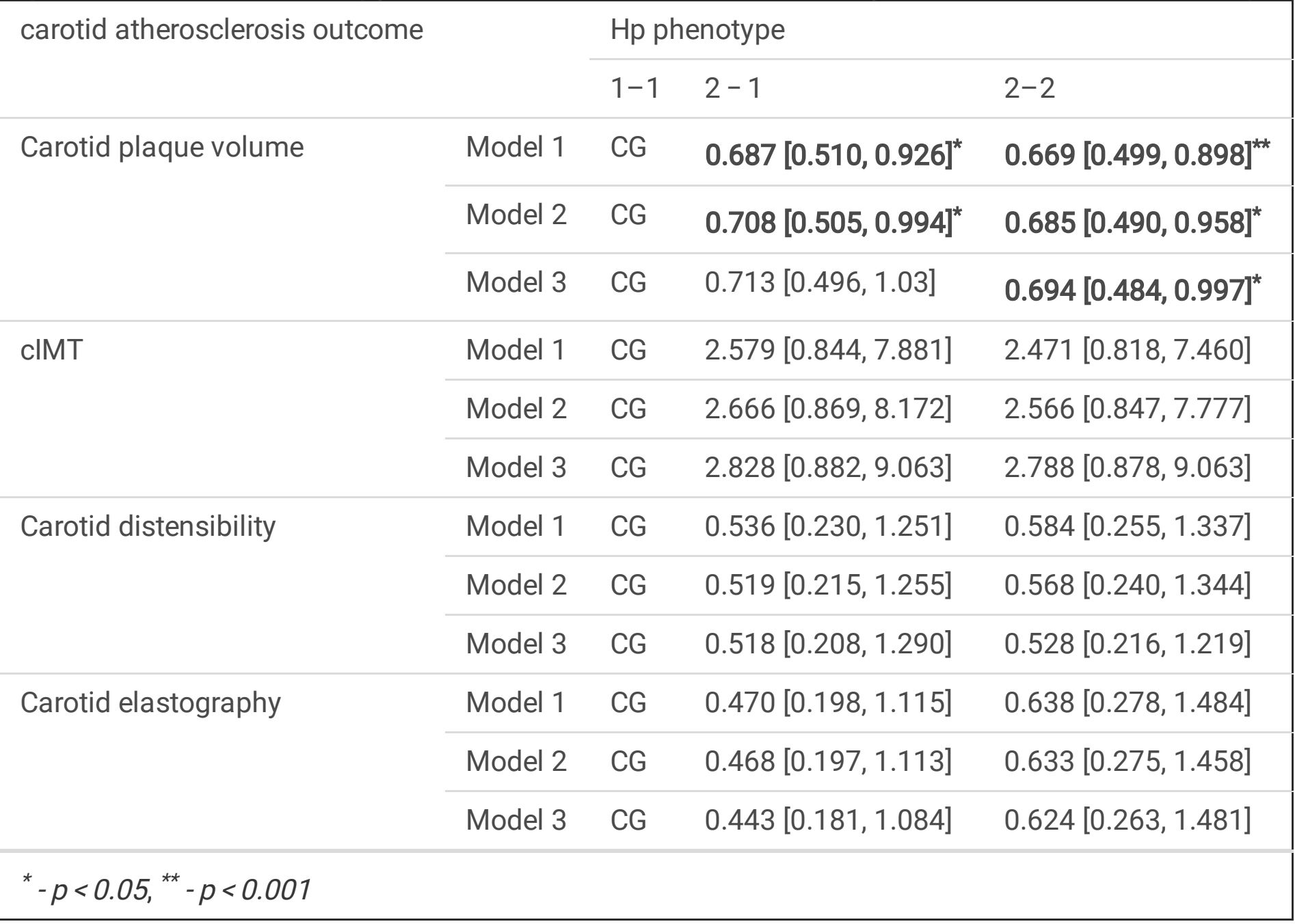

Ordinal regression demonstrated an approximate 30\% lower risk for higher plaque volume group for Hp 2 - 1 and Hp 2-2 phenotypes compared with Hp 1-1 phenotype (OR: 0.687 [0.510, 0.926], 0.669 [0.499, 0.898 ] for $\mathrm{Hp} 2-1, \mathrm{Hp} 2-2$ respectively). This relationship persisted after adjustment to age and gender (OR: 0.708 [0.505, 0.994], 0.685 [0.490, 0.958] for $\mathrm{Hp} 2-1, \mathrm{Hp} 2-2$ respectively) and cardiovascular risk factors (OR: 0.713 [0.496, 1.03], 0.694 [0.484, 0.997] for Hp 2 - 1, Hp 2-2 respectively). Other carotid atherosclerosis outcomes were not significantly associated with $\mathrm{Hp}$ phenotype, although the same trend for impaired carotid distensibility was demonstrated (OR: 0.518 [0.208, 1.290], $0.528[0.216,1.219]$ for Hp $2-1$, Hp 2-2 respectively) and carotid elastography (OR: 0.443 [0.181, 1.084], $0.624[0.263,1.481]$ for Hp $2-1$, Hp 2-2 respectively). Impaired cIMT also was also not significantly associated with Hp phenotype, yet showed an opposite relation (OR: 2.828 [0.882, 9.063], 2.788 [0.878, 9.063] for Hp 2 - 1, Hp 2-2 respectively).

\section{Discussion}


In this study of elderly non-demented type 2 diabetes patients, we found a significant association between greater number of $\mathrm{Hp}-1$ alleles with a larger volume of carotid plaques. This observation persisted when adjusting for numerous cardiovascular risk factors and diabetes related covariates. We found that $\mathrm{Hp} 2$ carriers have a $30 \%$ lower risk for larger carotid plaque volume compared to $\mathrm{Hp} 1-1$ carriers. These results are consistent with recent literature of associations of $\mathrm{Hp} \mathrm{1-1}$ with cerebrovascular disease ${ }^{8,25}$. In contrast, we did not find significant associations between $\mathrm{Hp}$ phenotype and cIMT, carotid distensibility and elastography measures.

Our results together with those of others ${ }^{8,9}$ suggest that $\mathrm{Hp}$ may serve different functions in different vascular beds depending on the underlying pathophysiological processes. Among type 1 diabetes patients, haptoglobin 1-1 is associated with protection against coronary artery disease but with a greater risk for stroke. Furthermore, Type 1 diabetes individuals carrying the Hp 1-1 genotype had a greater volume of cerebral white matter hyperintensities ${ }^{8}$. Similarly, among hypertensive patients a correlation between Hp1-1 phenotype and the extent of hypertensive deep white matter damage has been shown ${ }^{9}$. The potential deleterious role of $\mathrm{Hp} \mathrm{1-1}$ in the brain is also supported by our prior findings of poorer cognitive function in $\mathrm{Hp}$ 1-1 genotype carriers ${ }^{25}$ and smaller hippocampal volume in $\mathrm{Hp}$ 1-1 carriers with poor glycemic control in the IDCD study ${ }^{26}$. There is preliminary data that $\mathrm{Hp}-1$ allele has poorer regeneration after endothelial injury ${ }^{27,28}$, suggesting a different mechanism of haptoglobin in the coronary arteries and the brain. Moreover, type 2 diabetes patients with Hp 2-2 phenotype were at greater risk of thickened cIMT and with unstable carotid plaque ${ }^{3,5}$, yet this is the first study to systematically investigate the association between the $\mathrm{Hp}$ phenotypes and carotid plaque volume. Whether $\mathrm{Hp}$ phenotypes have different pathogenic roles on carotid plaque volume, plaque composition and plaque location, requires further investigation.

Although the associations of Hp phenotype with carotid elastography and distensibility were not significant, their trend was similar to that of plaque volume, i.e. $\mathrm{Hp} \mathrm{1-1} \mathrm{carriers} \mathrm{had} \mathrm{poorer} \mathrm{elastography}$ and distensibility. Quantification of plaque burden is superior to cIMT because the plaque burden measures and their progression have been shown to strongly predict CVD events and can identify high risk patients ${ }^{29}$ whereas meta-analysis results showed that cIMT without plaque thickness is a weak predictor of CVD risk ${ }^{30}$ and its change over time does not predict CVD events ${ }^{31}$.

The study has several limitations that deserve consideration. There might be a survival bias where one of the phenotypes is less represented because of early drop out due to morbidity or mortality or healthy patient selection bias. For example, the $\mathrm{HbA} 1 \mathrm{c}$ in the subsample that participated in the carotid assessments was $6.6 \pm 0.71 \%$, which is lower than that of the full baseline IDCD study ${ }^{18}$ (HbA1C $=6.9 \pm$ 0.74). The distribution of the haptoglobin phenotypes in this cohort was, however, consistent with the Hardy-Weinberg equation and similar to the distribution in Europe and Israel ${ }^{22,23}$. The study is cross sectional, and investigation of the associations of $\mathrm{Hp}$ with changes over time in carotid structure and function is warranted. Strengths of this study include a relatively large number of participants assessed with a state of the art carotid battery including volumetric measurement of carotid plaque, the well 
validated diagnosis of T2D, and the broad range of directly measured (rather than self-reported) covariates.

\section{Conclusion}

Our results suggest that among elderly patients with type 2 diabetes, the Hp phenotype is associated with atherosclerotic carotid plaque volume adding and broadening the evidence to the involvement of the $\mathrm{Hp}$ 1-1 phenotype in the pathophysiology of cerebrovascular disease.

\section{Abbreviations}

CCA - common carotid artery

CHD - coronary heart disease

$\mathrm{Cl}$ - confidence interval

cIMT - carotid Intima Media Thickness

CVD - cardiovascular disease

Hp - Haptoglobin

HMO - health maintenance organization

IDCD - Israel Diabetes and Cognitive Decline

MHS - Maccabi Healthcare Services

$\mathrm{OR}$ - odds ratio

$S R$ - strain ration

TIA - transient ischemic attack

VPQ - Vascular Plaque Quantification

$\mathrm{ROI}$ - region of interest

\section{Declarations}

1. Ethics approval and consent to participate - The study was approved by the Institutional Review Board of Mount Sinai and the Helsinki committees of Sheba and MHS.

2. Consent for publication - not applicable 
3. Availability of data and materials - The datasets used and/or analysed during the current study are available from the corresponding author on reasonable request.

4. Competing interests - The authors declare that they have no competing interests

5. Funding - This study was funded by grants from NIH grants AG053446, AG051545, AG034087 and AG043878 and donors of Alzheimer's Disease Research, a program of BrightFocus Foundation.

\section{Authors contribution -}

6.1 CBM - data collection, analysis and interpretation of data, drafted the work

6.2 SH - design of the work, interpretation of data

6.3 RRS - design of the work

6.4 AH - substantial contribution to the conception

6.5 AL - analysis and interpretation of data

6.6 MSB - substantial contributions to the conception, design of the work, interpretation of data, substantively revised the work

6.7 DT - substantial contributions to the conception, design of the work, interpretation of data, substantively revised the work

7. Acknowledgements - to the donors of Alzheimer's Disease Research, a program of BrightFocus Foundation, for support of this research and NIH grants AG053446, AG051545, AG034087 and AG043878.

\section{Acknowledgments}

Acknowledgement is made to the donors of Alzheimer's Disease Research, a program of BrightFocus Foundation, for support of this research and NIH grants AG053446, AG051545, AG034087 and AG043878, and the LeRoy Schecter Foundation.

\section{References}

1. Levy AP, Asleh R, Blum S, et al. Haptoglobin: Basic and Clinical Aspects. 2010;12(2).

2. Adams JN, Cox AJ, Freedman BI, Langefeld CD, Carr JJ, Bowden DW. Genetic analysis of haptoglobin polymorphisms with cardiovascular disease and type 2 diabetes in the diabetes heart study. Cardiovasc Diabetol. 2013;12(1):31. doi:10.1186/1475-2840-12-31.

3. Dalan R, Liew H, Goh LL, et al. The haptoglobin 2-2 genotype is associated with inflammation and carotid artery intima-media thickness. Diabetes Vasc Dis Res. 2016;13(5):373-6. 
doi:10.1177/1479164116645247.

4. Roguin A, Ribichini F, Ferrero V, et al. Haptoglobin Phenotype and the Risk of Stent Implantation. J Cardiol. 2002;89(02):806-10.

5. ljäs P, Saksi J, Soinne L, et al. Haptoglobin 2 allele associates with unstable carotid plaque and major cardiovascular events. Atherosclerosis. 2013;230(2):228-34.

doi:10.1016/j.atherosclerosis.2013.07.008.

6. Levy AP, Hochberg I, Jablonski K, et al. Haptoglobin phenotype is an independent risk factor for cardiovascular disease in individuals with diabetes: The strong heart study. J Am Coll Cardiol. 2002;40(11):1984-90. doi:10.1016/S0735-1097(02)02534-2.

7. Levy AP, Larson MG, Corey D, Lotan R, Vita JA, Benjamin EJ. Haptoglobin phenotype and prevalent coronary heart disease in the Framingham offspring cohort. Atherosclerosis. 2004;172(2):361-5. doi:10.1016/j.atherosclerosis.2003.10.014.

8. Costacou T, Rosano $\mathrm{C}$, Aizenstein $\mathrm{H}$, et al. The haptoglobin 1 allele correlates with white matter hyperintensities in middle-aged adults with type 1 diabetes. Diabetes. 2015;64(2):654-9. doi:10.2337/db14-0723.

9. Staals J, Henskens L, Delanghe J, et al. Haptoglobin Phenotype Correlates with the Extent of Cerebral Deep White Matter Lesions in Hypertensive Patients. Curr Neurovasc Res. 2010;7(1):1-5. doi:10.2174/156720210790820163.

10. Staals J, Pieters BMA, Knottnerus ILH, Rouhl RPW, Oostenbrugge RJ van, Lodder JRD. and J. Haptoglobin Polymorphism and Lacunar Stroke. Curr Neurovasc Res. 2008;5(3):153-8. doi:http://dx.doi.org/10.2174/156720208785425675.

11. Kumar A, Yang EY, Brunner G, et al. Plaque Volume of Carotid Endarterectomy Specimens Measured by 3D Ultrasound Technology. JACC Cardiovasc Imaging. 2017;9(9):1118-9. doi:10.1016/j.jcmg.2015.10.007.

12. Quantification AP. Inter-Rater Reliability of Carotid Atherosclerotic Plaque Quantification by 3Dimensional Sonography. 2014:1273-1278. doi:10.7863/ultra.33.7.1273.

13. Inaba Y, Chen JA, Bergmann SR. Carotid plaque, compared with carotid intima-media thickness, more accurately predicts coronary artery disease events : A meta-analysis. Atherosclerosis. 2012;220(1):128-33. doi:10.1016/j.atherosclerosis.2011.06.044.

14. Wannarong T, Parraga G, Buchanan D, et al. Cardiovascular Events. 2013:1859-65. doi:10.1161/STROKEAHA.113.001461.

15. Sillesen H, Sartori S, Sandholt B, Baber U, Mehran R, Fuster V. Carotid plaque thickness and carotid plaque burden predict future cardiovascular events in asymptomatic adult Americans. Eur Heart $\mathrm{J}$ Cardiovasc Imaging. 2018;19(9):1042-50. doi:10.1093/ehjci/jex239.

16. van Sloten TT, Stehouwer CDA. Carotid Stiffness: A Novel Cerebrovascular Disease Risk Factor. Pulse. 2016;4(1):24-7. doi:10.1159/000445354.

17. Van Sloten TT, Sedaghat $S$, Laurent $S$, et al. Carotid Stiffness Is Associated with Incident Stroke A Systematic Review and Individual Participant Data Meta-Analysis. J Am Coll Cardiol. 
2015;66(19):2116-25. doi:10.1016/j.jacc.2015.08.888.

18. Beeri MS, Ravona-Springer R, Moshier E, et al. The Israel Diabetes and Cognitive Decline (IDCD) study: Design and baseline characteristics. Alzheimer's Dement. 2014;10(6):769-78. doi:10.1016/j.jalz.2014.06.002.

19. No Title. www.icd9data.com/2007/Volume1..

20. Chodick G, Heymann AD, Shalev V, Kookia E. The Epidemiology of Diabetes in a Large Israeli HMO. Eur J Epidemiol. 2003;18(12):1143-6. http://www.jstor.org/stable/3582888.

21. Ravona-Springer R, Heymann A, Schmeidler J, et al. Trajectories in Glycemic Control over Time Are Associated with Cognitive Performance in Elderly Subjects with Type 2 Diabetes. PLoS One. 2014;9(6):e97384. doi:10.1371/journal.pone.0097384.

22. Hochberg I, Roguin A, Nikolsky E, Chanderashekhar PV, Cohen S, Levy AP. Haptoglobin phenotype and coronary artery collaterals in diabetic patients. Atherosclerosis. 2002;161(2):441-6. doi:10.1016/S0021-9150(01)00657-8.

23. Shor M, Boaz M, Gavish D, Wainshtein J. Relation of Haptoglobin Phenotype to Early Vascular Changes in Patients With Diabetes Mellitus. 2007. doi:10.1016/j.amjcard.2007.07.052.

24. Chemistiy C. Biological and clinical significance of haptoglobin polymorphism. 1996;1600:15891600.

25. Guerrero-Berroa E, Ravona-Springer R, Heymann A, et al. Haptoglobin genotype modulates the relationships of glycaemic control with cognitive function in elderly individuals with type 2 diabetes. Diabetologia. 2015;58(4):736-44. doi:10.1007/s00125-014-3487-2.

26. Livny A, Ravona-Springer R, Heymann A, et al. Haptoglobin 1-1 Genotype Modulates the Association of Glycemic Control With Hippocampal Volume in Elderly Individuals With Type 2 Diabetes. Diabetes. 2017;66(11):2927-32. doi:10.2337/db16-0987.

27. Rouhl R, Oostenbrugge R, Damoiseaux JG, et al. Haptoglobin Phenotype May Alter Endothelial Progenitor Cell Cluster Formation in Cerebral Small Vessel Disease. Curr Neurovasc Res. 2009;6(1):32-41. doi:10.2174/156720209787466082.

28. Rouhl RPW, Mertens AECS, Van Oostenbrugge RJ, et al. Angiogenic T-cells and putative endothelial progenitor cells in hypertension-related cerebral small vessel disease. Stroke. 2012;43(1):256-8. doi:10.1161/STROKEAHA.111.632208.

29. Wannarong T, Parraga G, Buchanan D, et al. Progression of Carotid Plaque Volume Predicts Cardiovascular Events. Stroke. 2013;44(7):1859. http://stroke.ahajournals.org/content/44/7/1859.abstract. LP - 1865.

30. Hester MD, Ruijter, Anderson TJ, Britton AR, Jacqueline M, et al. Common Carotid Intima-Media Thickness Measurements in Cardiovascular Risk Prediction. J Am Med Assoc. 2015;308(8):796803. http://jama.jamanetwork.com/.

31. Lorenz MW, Polak JF, Kavousi M, et al. Carotid intima-media thickness progression to predict cardiovascular events in the general population (the PROG-IMT collaborative project): A meta- 
analysis of individual participant data. Lancet. 2012;379(9831):2053-62. doi:10.1016/S01406736(12)60441-3.

32. Heymann AD, Chodick G, Halkin $H$, et al. The implementation of managed care for diabetes using medical informatics in a large Preferred Provider Organization. Diabetes Res Clin Pract. 2006. doi:10.1016/j.diabres.2005.07.002.

Figures

\section{1 active participants at 36 months FU (FU2) at the IDCD}

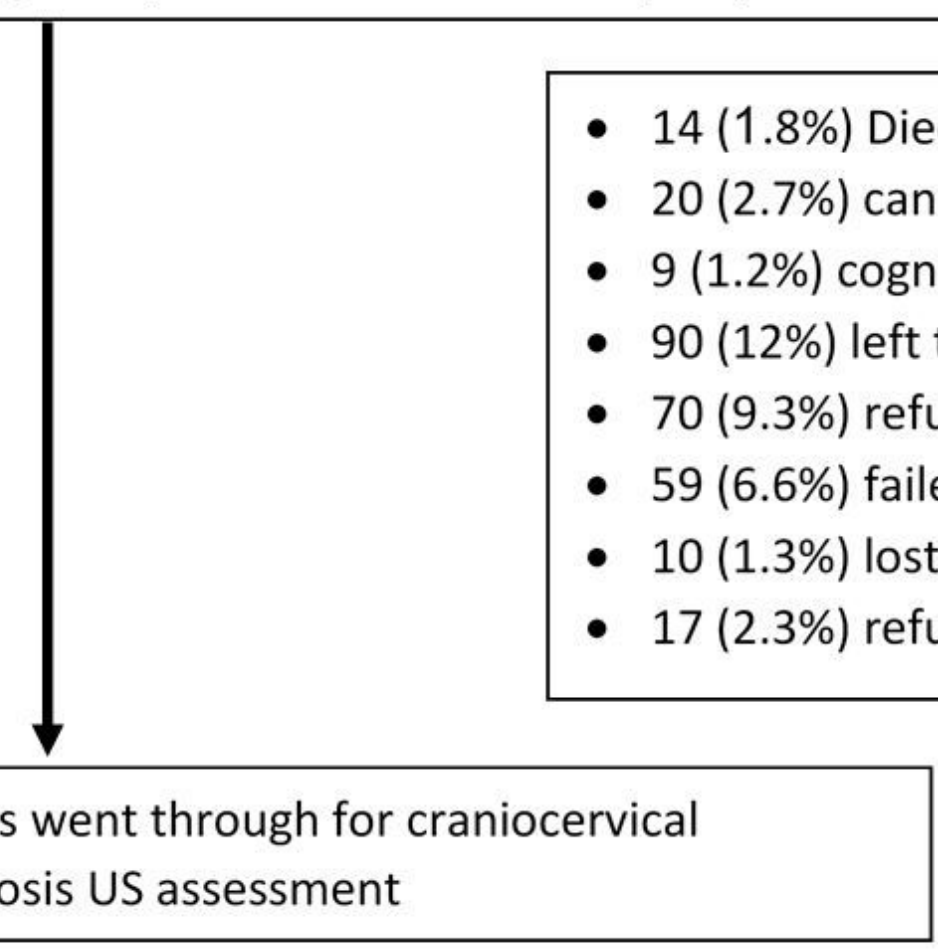

472 patients went through for craniocervical atherosclerosis US assessment

Figure 1

Flow chart of the patients in the IDCD carotid cohort 


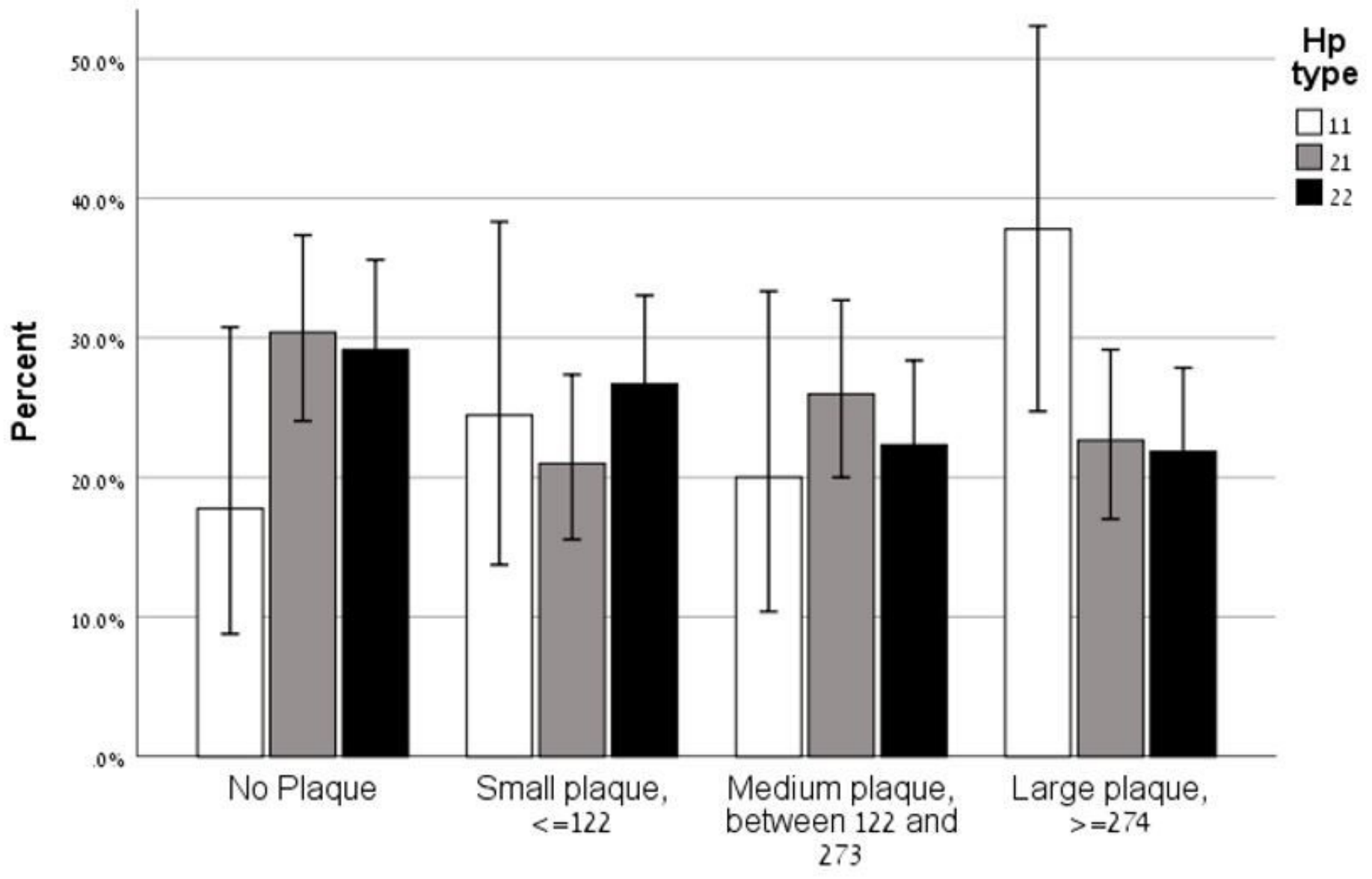

PlaqueVol_cat

Error bars: $95 \% \mathrm{Cl}$

Figure 2

Distribution of Hp phenotype group by Plaque volume group. 